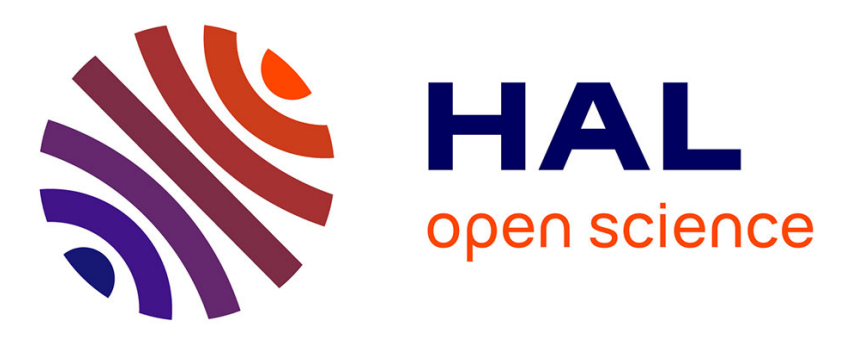

\title{
An SDN Approach to Adaptive Video Streaming in Wireless Home Networks
}

Rabee Mustapha Abuteir, Anne Fladenmuller, Olivier Fourmaux

\section{To cite this version:}

Rabee Mustapha Abuteir, Anne Fladenmuller, Olivier Fourmaux. An SDN Approach to Adaptive Video Streaming in Wireless Home Networks. The International Wireless Communications \& Mobile Computing Conference (IWCMC 2016), Sep 2016, Paphos, Cyprus. hal-01365347

\section{HAL Id: hal-01365347 https://hal.sorbonne-universite.fr/hal-01365347}

Submitted on 13 Sep 2016

HAL is a multi-disciplinary open access archive for the deposit and dissemination of scientific research documents, whether they are published or not. The documents may come from teaching and research institutions in France or abroad, or from public or private research centers.
L'archive ouverte pluridisciplinaire HAL, est destinée au dépôt et à la diffusion de documents scientifiques de niveau recherche, publiés ou non, émanant des établissements d'enseignement et de recherche français ou étrangers, des laboratoires publics ou privés. 


\title{
An SDN Approach to Adaptive Video Streaming in Wireless Home Networks
}

\author{
Rabee Mustapha Abuteir, Anne Fladenmuller and Olivier Fourmaux \\ Sorbonne Universités, UPMC Univ Paris 06, CNRS, LIP6 UMR 7606, 4 place Jussieu 75005 Paris \\ Email: \{mustapha.abuteir, anne.fladenmuller, olivier.fourmaux\}@lip6.fr
}

\begin{abstract}
Adaptive video streaming techniques were introduced to enable high quality video delivery over HTTP. These schemes propose to choose dynamically the appropriate video rate to match the operating conditions. In home networks, wireless access is the predominant Internet access. Multiple clients/players with different link qualities compete over a limited wireless bandwidth to transfer their video streams. As a result, some users undergo unpredictable degradations of their Quality of Experience (QoE) while others benefit from these perturbations. In this paper we introduce a new technique to address this issue at the gateway without modifying neither the client nor the video server side. We design a framework WNAVS (Wireless Network Assisted Video Streaming) that relies on the deployment of Software Defined Networking (SDN). WNAVS performs a dynamic traffic shaping based on collected network traffic statistics and allocates bandwidth for the clients in real time. We evaluate WNAVS over several metrics: fairness, instability, average video quality as well as the video traffic utilization. Our results demonstrate an improvement for all these parameters.
\end{abstract}

Index Terms-DASH, SDN, TCP, Traffic shaping, Video streaming, Wireless home networks.

\section{INTRODUCTION}

Internet video is the dominant Internet traffic, last report from Cisco Systems [1] shows the Internet video traffic reaches $59 \%$ of the total Internet traffic in 2014 and it should reach $77 \%$ by 2019 . The same report expects the WiFi and mobile traffic to account $66 \%$ of the total Internet traffic by 2019 . This tremendous increase in wireless Internet and video usage raise new challenges to maintain users satisfaction while operating conditions may experience strong variations. Dynamic Adaptive Streaming over HTTP (DASH) [2] is an ISO standard also known as MPEG-DASH. It was developed as a cooperation between industries and standards organizations to provide high quality video delivery and increase the user Quality of Experience (QoE). Adaptive video schemes propose to divide the video into chunks and make them available at different bitrates at the server side. The client (player) defines the next chunk bitrate but the way it is chosen is kept open to the implementer. When 2 or more DASH players compete for a limited bandwidth [3] problems may raise leading to instabilities in resource allocation, unfairness between the players requested bitrates and network bandwidth under-utilization.

In wireless home networks, wireless could cause bottleneck [4] [5]. In addition, the wireless throughput could be effected by many factors such as the number of clients, number of neighbor wireless networks, interference with non-WiFi

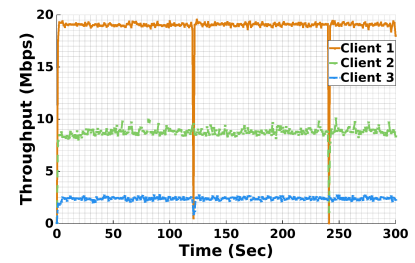

(a) Three clients run individually.

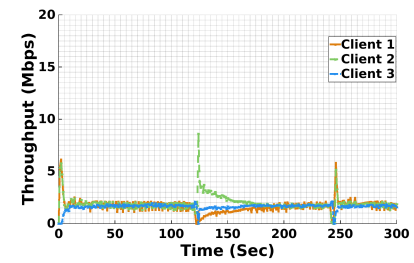

(b) Three clients run simultaneously.
Fig. 1: Instantaneous TCP throughput for three clients. We used the same topology and parameters in section IV.

devices and distance between the client and the gateway ${ }^{1}$. Meanwhile, DASH runs over HTTP which uses TCP as transport layer, Thus lead to mismatch between the DASH adaptation logic which runs at the client side and the TCP congestion control which runs at server side. When multiple clients stream videos over HTTP with different links qualities (one or more with poor link quality) the TCP performance decline. Fig. 1 shows the TCP performance for three clients with different links qualities. In the first scenario only one client run per time, The TCP throughput vary due to the variation in links qualities. In the second scenario the three clients run together, the TCP throughput depressed. This TCP performance issue happened because the wireless channel is shared, TCP fairness and performance anomaly of IEEE 802.11 networks [6] [7]. Hence, the low TCP throughput and the competition between the players will lead to bad QoE.

Software-Defined Networking (SDN) [8] is a network architecture aims to decouple the control and data plane. In this paper we proposed a new framework WNAVS (Wireless Network Assisted Video Streaming) which is built on the top of SDN. WNAVS aims to enhance the TCP performance, stream videos in higher bitrates and increase the users QoE. In Addition, WNAVS reduces players instability, maximizes the fairness between clients and increases the videos qualities streamed by the clients.

The rest of the paper is organized as follows. Related work are presented in Section II. In Section III we explain our proposed framework (WNAVS). The performance evaluation is presented in section IV. Finally, the conclusion and future work is discussed in Section V.

\footnotetext{
${ }^{1}$ In this paper wireless home gateway, gateway and access point refer to the same thing.
} 


\section{RELATED WORK}

DASH protocol behaviour over Internet leads to suboptimal performance due to: (1) the adaptation algorithm performance depends on the chunks duration (2) the disparity between the advertised chunk quality and real encoding rate (3) the dependence on HTTP/TCP to deliver the video streaming and the ON-OFF pattern of video streaming lead to inefficient bandwidth utilization [3]. In this paper we focus on the third cause which is related to the network behavior and it is common in wireless home networks.

Airtime fairness [9] was proposed as a solution for 802.11 anomaly (client with bad link quality decreases throughput for the other clients as showed in Fig. 1). Airtime fairness resolves the anomaly problem with uplink traffic but the majority of traffic in video streaming is downlink to the clients through the gateway. The authors in [10] proposed a scheduler at the Access Point (AP) to picks only a subset of clients to be served by the AP during any instant. This technique is not suitable for delay sensitive application such as video streaming. HTTP Adaptive Streaming (HAS) traffic shaping to improve users QoE in home networks was proposed in [11] [12]. However, in wireless networks the link quality vary from client to other and it should be taken into account. Ramamurthi et al [13], introduced a link awareness adaptation logic to make the application layer response to change in wireless link faster. The anomaly behaviour will reduce the TCP throughput and that leads to bad QoE.

In [14], the authors proposed a heuristic cross-layer adaptation logic to enhance UDP video streaming in WiFi environment but the majority of Internet run over TCP. Chiariotti et al [15], proposed a centralize QoE aware variable bitrate streaming. WNAVS framework is different because it keeps both the clients and the server side without change. A proxy based solution was proposed in [16], the proxy fetches segments and redirects the requests based on channel prediction. This technique is inconsistent with the control loop between the players and the OTT server. In [17], a dynamic rate adaptation algorithm for wireless networks was proposed. The algorithm collected bandwidth statistics and chooses the optimal rate for the clients. When there is bottleneck in wireless access the optimal rate will be low and that decease the QoE. Chenji et al [18], proposed a bandwidth allocation algorithm to increase the QoE and reduce the bandwidth utilization. However, this technique will not work with video streaming due to large bandwidth demand of video streams, the competition between player and TCP behavior in wireless networks. In-network QoE measurement framework based on SDN architecture was proposed in [19]. This framework collects QoE parameters for video streams to assist content distribution services or network management. $\mathrm{Mu}$ et al [20] proposed SDN framework for user level fairness but the variations in links qualities between the clients aren't taken into account. Mansy et al [21] proposed a Video Home Shaper (VHS) to improve video streaming for home networks. The variation in links qualities between the clients wasn't taken into account.

In WNAVS we used SDN architecture because we believe in near future we will have more devices in wireless home networks and the best way to manage the network is through SDN. This vision is consistent with trends of future home networks [22]. WNAVS shape the traffic at the gateway, both the clients and the server side is kept without change. WNAVS improves the QoE by increasing the TCP throughput in wireless networks and reduces the competition between video flows. We will discuss the proposed framework in more details in the next section.

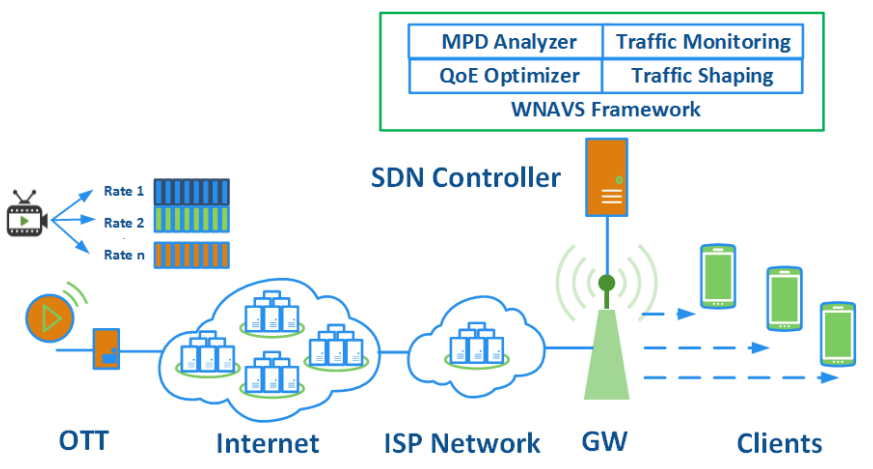

Fig. 2: Video streaming in wireless home networks using WNAVS framework.

\section{PRoposed FrameWORK}

In wireless home networks, multiple clients accesses the Internet through the gateway. The bottleneck could happen in the access link or in the wireless link [23]. In [24] we proposed a solution to improve video streaming when there is bottleneck in the access link. When the wireless links qualities between the clients and the gateway vary the throughput decreases as showed in Fig. 1. In this paper we propose a solution to enhance the QoE of video streaming when the bottleneck is in the wireless link. We build WNAVS (Wireless Network Assisted Video Streaming) based on SDN architecture. WNAVS uses dynamic traffic shaping to allocates bandwidth, reduces the contention and increases TCP throughput. Subsequently, using WNAVS will lead to better QoE for the users.

Fig. 2 shows a typical architecture for video streaming in wireless home networks. We have multiple clients with different wireless links qualities connected to the gateway. The gateway is connected to the Internet through the ISP network and it managed by the SDN controller. The videos are available at the OTTs with different bitrates and the clients stream this videos using DASH. WNAVS framework run on the top of the SDN controller to improve the users QoE. We build WNAVS as a modular framework so any future upgrade could be easily integrated. In addition, we used an abstract model for the controller so its location is less important than its function. The controller consists from four modules and next we will describe each one briefly. 


\section{A. Traffic Monitoring Module}

This module is responsible for flows classification and monitoring. When a new traffic flow starts, the first packet reaches the gateway that forwards it to the controller. Then the controller classifies the flows (e.g, using DNS based classification [25]). Further, the gateway sends periodically statistics to the controller. When the controller doesn't receive statistics about a flow for certain period that means is not active any more. The gateway sends statistics periodically to the controller and one of these statistics is the effective wireless rate [26]. Wireless effective rate is a metric reflects the link quality and packet losses for each clients. Notably, when the link quality is good between the client and the gateway, the effective wireless rate is high and vice versa. The wireless effective rate is defined for client $n$ as :

$$
E_{n}=\frac{1}{p_{n}} \sum_{1}^{i} s_{n i} m_{i}
$$

Where $p_{n}$ is the total packets for the client $n, i$ is the number of physical transmission rate could be used between the client and the gateway (e.g., for 802.11g $1 \leq i \leq 8$ ), $s_{n i}$ is the number successfully packet for client $n$ at physical rate $m_{i}$.

\section{B. MPD Analyzer Module}

This module is used to analyze the Media Presentation Description (MPD) file. This file is downloaded from the OTT server by the player and it contains metadata about the video. When this file reaches the gateway, a copy is forwarded to the controller. The MPD file contains the video length, the number of chunks, the available bitrates and the chunks URLs. The statistics collected from the gateway and the MPD metadata for different video streams will be used by the QoE optimizer to allocates bandwidth for the clients.

\section{QoE Optimizer Module}

When we have multiple clients stream videos using DASH from OTT servers, since DASH run over HTTP which uses TCP as transport protocol, the wireless link could be a bottleneck if one more clients have bad link quality. Hence, the wireless throughput decreases due to the performance anomaly of IEEE 802.11 and TCP fairness. The main cause of performance anomaly is the clients with bad links qualities will slow down the clients with good links qualities. Consequently, the TCP throughput will decrease due to the TCP fairness. To solve this problem we propose a fair link sharing between the clients, so each client will get bandwidth proportionally to its link quality. We choose to implement our solution using traffic shaping because it is widely supported by real wireless home gateways [27] [28].

To increase the aggregate TCP throughput we need to know the maximum achievable throughput by each client individually. We use the same model used in [26]. The authors found the maximum value of correlation coefficient between the Link Experience (LE) and The TCP throughput over many metrics. The link experience for client $n$ is defined as:

$$
L E_{n}=(1-a)(1-c) E_{n}
$$

Where $a$ is the air time utilization from other sources, $c$ local contention. Since we will evaluate our work in simulation there is no air time utilization from other sources. We need to reduce the contention between clients to maximize the aggregate TCP throughput. Since we have $N$ clients, we need each client to have the same contention probability, we should set $c=\frac{1}{N}$. Hence, the new expression link experience is:

$$
L E_{n}=\frac{1}{N} E_{n}
$$

Based on [26] the WiFi TCP throughput (Witt) for client $n$ is calculated using this expression:

$$
\text { Witt }_{n}=\beta_{1} L E_{n}+\beta_{0}
$$

Where $\beta_{1}$ and $\beta_{0}$ are constants, their values depend on the IEEE 802.11 standard (e.g., for IEEE $802.11 \mathrm{~g}$ they are 0.422 and 0.167 [26]). The effective wireless rate is collected by the traffic monitoring module periodically. Then the gateway shape the traffic for each client $\mathrm{n}$ to bandwidth $B_{n}$ and it is defined as :

$$
B_{n}= \begin{cases}W i t t_{n}, & \text { if } W_{i t t}>(1+C) R_{\text {min }} . \\ (1+C) R_{\text {min }}, & \text { otherwise. }\end{cases}
$$

Where $C$ is the DASH protocol overhead ${ }^{2}, R_{\min }$ is the minimum video bitrate available at the server and is extracted by the MDP analyzer. We use equation 5 to prevents clients with bad links qualities from starvation. The QoE optimizer calculates the bandwidth that should assign to the clients periodically. Using this techniques we evaluate the fair TCP throughput should be assigned to each client proportionally to its link quality. This technique will increase the aggregate wireless throughput since it reduce the performance anomaly of IEEE 802.11 networks. The video flow becomes inactive due to the stop of video session or the ON-OFF pattern of adaptive video streaming when the buffer is full. For ONOFF pattern of video streaming, WNAVS will increase the bandwidth for each client and it make sure the new bandwidth is less then $(1+C) R_{N}$. Where $R_{N}$ is the next bitrate higher than the current bitrate used by the client. Thus, WNAVS increase the wireless throughput without decreasing the video streams instability. If the video session stopped, WNAVS removes the inactive flow and recalculate the bandwidth used for the clients.

\section{Traffic Shaping Module}

This module is informed about the bandwidth should be allocated to each client. These values are proportional to the clients links qualities. Then the traffic shaping module

\footnotetext{
${ }^{2}$ When a client streams a video at bitrate $\mathrm{R}$ it need bandwidth $(1+C) R$. In our evaluation we used $\mathrm{C}=0.3$. For farther details you can refer to our paper in [24].
} 
apply these values at the gateway. WNAVS uses dynamic traffic shaping because the bandwidth allocation for each client depends on the variable number of video flows and wireless links qualities between the clients and the gateway.

\section{Evaluation AND RESUlts}

This section is divided into two subsections. In the first subsection the performance metrics used to evaluate WNAVS are presented. In the second subsection we evaluate the performance of WNAVS.

\section{A. Performance Metrics}

To evaluate WNAVS we choose four metrics widely used in the literature reflects the QoE for video streaming.

1) Fairness: It is based on Jain fairness [29] which is widely used in literature to evaluate the fairness between competing video streams [24] [21]. We define fairness between video streams as follow:

$$
\text { Fairness }=\frac{1}{T} \sum_{t=1}^{T} \frac{\left(\sum_{n=1}^{N} \frac{R_{n t}}{E_{n t}}\right)^{2}}{N \sum_{n=1}^{N}\left(\frac{R_{n t}}{E_{n t}}\right)^{2}}
$$

Where $T$ is the streaming session duration, $N$ is the total number of video streams, $R_{n t}$ is the video bitrate streamed by client $n$ at time $t$ and $E_{n t}$ is the wireless effective rate used by client $n$ at time $t$. The objective of this metric is to find the fairness between the bitrates and the wireless effective rates used to download it.

2) Instability: The QoE of DASH based video streams is extremely impacted by the bitrate switches [3] [21] [24]. The instability of video streams is defined as follow:

$$
\text { Instability }=\sum_{n=1}^{N} \frac{S_{n}}{T C_{n}}
$$

Where $S_{n}$ is the number of switches in bitrate for video stream $n$ and $T C_{n}$ is the total number of chunks downloaded for client $n$. Smaller instability reflects better performance and vice versa.

3) Average Video Quality $(A V Q)$ : The video quality increases as the bitrate increases [3] [24]. Higher average video quality means better QoE. The $A V Q$ is defined as follow:

$$
A V Q=\frac{1}{N T} \sum_{n=1}^{N} \sum_{t=1}^{T} R_{n t}
$$

4) Video Traffic Utilization (VTU): It is a fraction between the summation of bitrates used by video flows to the maximum wireless throughput (e.g, for IEEE 802.11g is 54mbps) [24]. The $V T U$ is defined as follow:

$$
V T U=\frac{1}{T} \sum_{t=1}^{T} \frac{\sum_{n=1}^{N} R_{t n}}{B W}
$$

Where $B W$ is the maximum wireless throughput. When video streams bitrates increase the video traffic utilization increases and vice versa.

TABLE I: Simulation Parameters

\begin{tabular}{c||c}
\hline \multicolumn{1}{c||}{ Simulation Parameters } & Value \\
\hline \hline WiFi Standard & IEEE 802.11g \\
\hline WiFi Rate Adaptation Algorithm & Minstrel \\
\hline DASH Adaptation Logic & Buffer Based and Smooth \\
& Adaptation \\
\hline Video Bitrates & $235,375,560,750,1050$, \\
& 80 seconds \\
\hline Player Buffer Size & 8 seconds \\
\hline Chunk Duration & 300 seonds \\
\hline Simulation Time &
\end{tabular}

\section{B. WNAVS Evaluation}

We implemente WNAVS framework in NS3 simulator [30]. We used the simulation parameters listed in table I. At the time of this work, NS3 didn't include any module for DASH video streaming. However, we have implemented a new module for NS3 to simulate the DASH video streaming. We encoded and open source film (Big Buck Bunny [31]) with the same bitrates used by Netflix [21] using FFmpeg [32]. Then we segmented the files to chunks using GPAC [33]. These chunks files were used in our simulation to be streamed over the network. To make the experiment more realistic we add background traffic so one client is downloading a file from a sink node. We run the simulation multiple times with different number of concurrent video streams ranging from 1 to 8 . The clients where distributed over the gateway coverage area with different links quality. We implement two rate adaptation algorithm widely used in literature, Smooth Adaptation (SA) [34] and Buffer Based Adaptation (BBA) [35]. WNAVS framework allocates the bandwidth at the gateway and it keeps the OTT server and the client side without modification. We compare the the performance metrics for both algorithm with and without WNAVS.

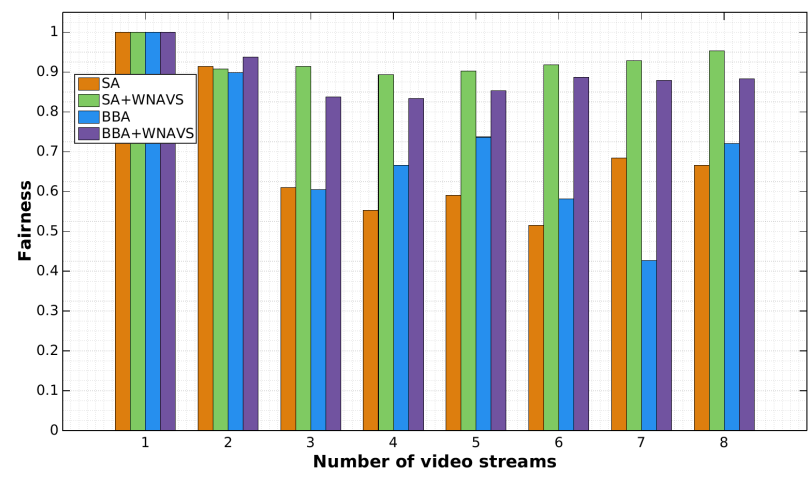

Fig. 3: Fairness between video streams with and without WNAVS framework. 
Fig. 3 shows the fairness vs the number of video streams. We compare both algorithm (SA and BBA) with and without WNAVS. The number of clients was varied from 1 to 8 with different links qualities. Both algorithm without WNAVS shows low fairness, thus happened because when a client stream video with bad link quality, it decrease the other clients throughput due to the anomaly behaviour of IEEE 802.11 networks. Hence, the TCP throughput of the video streams decrease due to the TCP fairness and that lead to channel under-utilization. In other hand, WNAVS framework shape the traffic for the clients proportionally to their links qualities using equation 5. Thus, the wireless throughput will increase due to the prevention of performance anomaly. As a result fairness between the clients will increase for both algorithm. In this paper, we choose the link quality fairness because it is common in wireless home networks to have clients with different link qualities.

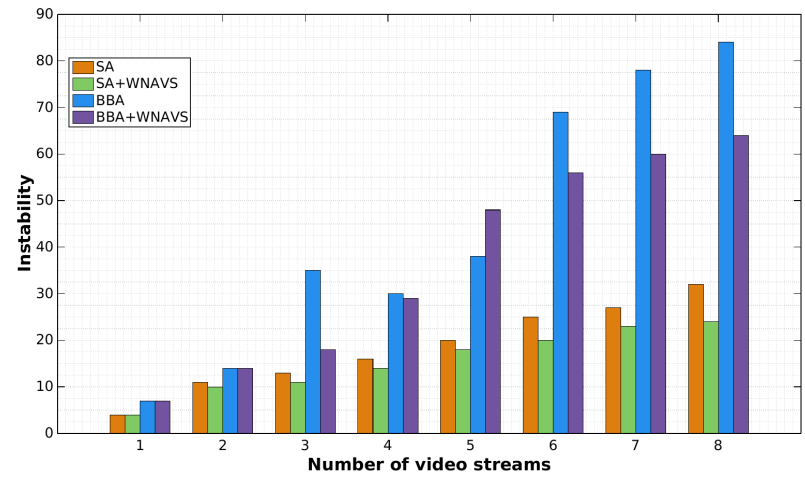

Fig. 4: Instability of video streams with and without WNAVS framework.

Fig. 4 shows the instability vs the number of video streams. When the number of video streams increase that lead to increase the competition between the players. The BBA algorithm chooses the next bitrate based on the buffer occupancy and that lead to higher instability compare to SA algorithm. The instability of players without WNAVS will increase due the contention in wireless networks and the depressed in TCP throughput. In the other hand, WNAVS shows more stability because of the dynamic bandwidth shaping. The clients will stream the video proportionally their links qualifies. Thus, reduces the instability of players and increase the QoE. Higher QoE will increase the clients engagement with the OTT video services.

Fig. 5 shows the average video quality vs the number of video streams. In wireless home networks when the number of clients increase the per-client throughput will decrease due to the share of wireless channel. For both algorithm without the assistance of WNVAS the average video qualities were low due to the depressed in TCP throughput. In comparison, WNAVS framework allocates bandwidth to the clients proportionally to their link qualities. This fairness between the clients eliminates the anomaly in the wireless access.

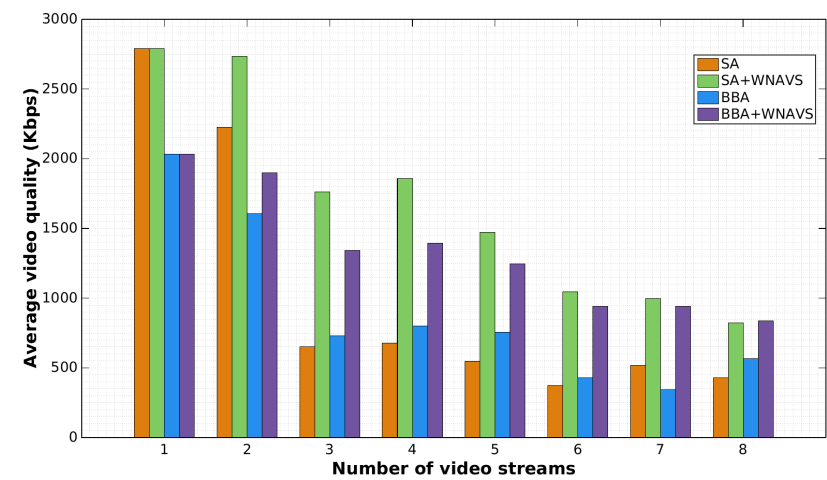

Fig. 5: Average video quality for video streams with and without WNAVS framework.

Consequently, the TCP throughput for clients with good link qualities will increase and that lead to stream video in higher rates and delivers the chunks faster. Using WANVS framework both algorithm show higher average video qualities which will increase the users QoE.

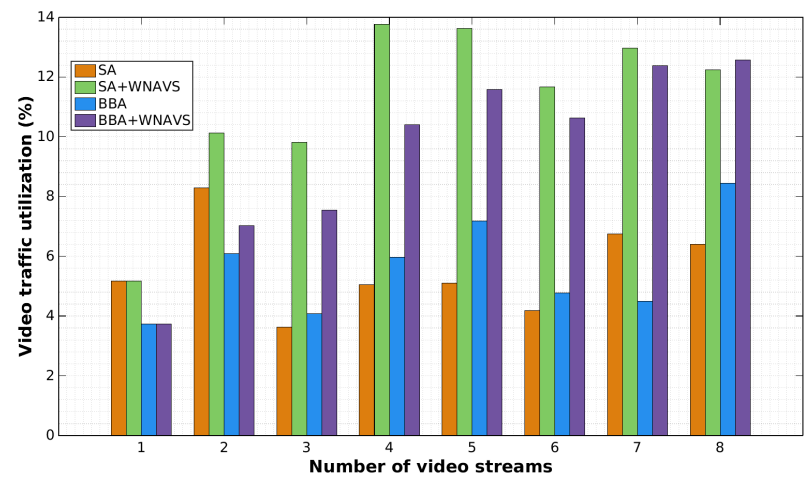

Fig. 6: Video traffic utilization of video streams with and without WNAVS framework.

Fig. 6 shows the video traffic utilization vs the number of video streams. When clients stream video without WNAVS framework, they stream with low TCP throughput and that cause low bitrates selection by the players. Thus, the video traffic utilization will decrease. Instead, WNAVS framework allocate bandwidth to the clients proportionally to their link quantities. Thus, the average video qualities increase and that lead to increase the video traffic utilization. Consequently, we manage the bandwidth in better way without changing neither the server nor the client side.

\section{COnClusion And Future Work}

We proposed WNAVS, a new SDN based framework to improve video streaming in wireless home networks. Our scheme is aimed to fairly share a wireless medium among various users and address the performance anomaly of IEEE 802.11 networks. This occurs when a transmission over a slow 
wireless link reduces the performance of all the other clients which are prevented to transmit concomitantly. Thus, WNAVS allocates bandwidth to the clients proportionally to their links qualities. When ON-OFF behaviour appears, WNAVS reallocates the bandwidth without causing instability for the players. WNAVS showed better performance for different QoE metrics for 2 adaptation algorithms widely used in the literature: SA (Smooth Adaptation) and BBA (Buffer Based Adaptation).

Our work will continue in the following directions. Firstly, we will use more complex QoE optimizer taking into account not only the link quality but also other parameters such as the screen resolution of the client [21]. Secondly, we will address the mobile video offloading from mobile operator to wireless home network and vice versa. Finally, WNAVS is the first step for Software Defined Home Networking (SDHN) and we will optimize it to be ready for implementation in commodity hardware.

\section{REFERENCES}

[1] C. Systems, "Visual networking index: Global ip traffic forecast 20142019," Feb,2016.

[2] V. Anthony and S. Iraj, "The mpeg-dash standard for multimedia streaming over the internet," IEEE Multimedia, no. 4, pp. 62-67, 2011.

[3] S. Bae, D. Jang, and K. Park, "Why is http adaptive streaming so hard?" in Proceedings of the 6th Asia-Pacific Workshop on Systems. ACM, 2015.

[4] I. Pefkianakis, H. Lundgren, A. Soule, J. Chandrashekar, P. Le Guyadec, C. Diot, M. May, K. Van Doorselaer, and K. Van Oost, "Characterizing home wireless performance: The gateway view," in Computer Communications (INFOCOM), 2015 IEEE Conference on. IEEE, 2015, pp. 2713-2731.

[5] S. Sundaresan, N. Feamster, and R. Teixeira, "Measuring the performance of user traffic in home wireless networks," in Passive and Active Measurement. Springer, 2015, pp. 305-317.

[6] S. Pilosof, R. Ramjee, D. Raz, Y. Shavitt, and P. Sinha, "Understanding tcp fairness over wireless lan," in INFOCOM 2003. Twenty-Second Annual Joint Conference of the IEEE Computer and Communications. IEEE Societies, vol. 2. IEEE, 2003, pp. 863-872.

[7] M. Heusse, F. Rousseau, G. Berger-Sabbatel, and A. Duda, "Performance anomaly of $802.11 \mathrm{~b}$," in INFOCOM 2003. Twenty-Second Annual Joint Conference of the IEEE Computer and Communications. IEEE Societies, vol. 2. IEEE, 2003, pp. 836-843.

[8] O. N. Fundation, "Software-defined networking: The new norm for networks," ONF White Paper, April,2012.

[9] P. Lin, W.-I. Chou, and T. Lin, "Achieving airtime fairness of delaysensitive applications in multirate ieee 802.11 wireless lans," Сотmunications Magazine, IEEE, vol. 49, no. 9, pp. 169-175, 2011.

[10] M. Maity, B. Raman, and M. Vutukuru, "Tcp download performance in dense wifi scenarios," in Communication Systems and Networks (COMSNETS), 2015 7th International Conference on. IEEE, 2015, pp. $1-8$.

[11] R. Houdaille and S. Gouache, "Shaping http adaptive streams for a better user experience," in Proceedings of the 3rd Multimedia Systems Conference. ACM, 2012, pp. 1-9.

[12] J. J. Quinlan, A. H. Zahran, K. Ramakrishnan, and C. J. Sreenan, "Delivery of adaptive bit rate video: balancing fairness, efficiency and quality," in Local and Metropolitan Area Networks (LANMAN), 2015 IEEE International Workshop on. IEEE, 2015, pp. 1-6.

[13] V. Ramamurthi, O. Oyman, and J. Foerster, "Using link awareness for http adaptive streaming over changing wireless conditions," in Computing, Networking and Communications (ICNC), 2015 International Conference on. IEEE, 2015, pp. 727-731.

[14] A. S. Abdallah and A. B. MacKenzie, "A cross-layer controller for adaptive video streaming over ieee 802.11 networks," in Communications (ICC), 2015 IEEE International Conference on. IEEE, 2015, pp. 67976802.
[15] F. Chiariotti, C. Pielli, A. Zanella, and M. Zorzi, "Qoe-aware video rate adaptation algorithms in multi-user ieee 802.11 wireless networks," in the Proceedings of the IEEE International Conference on Communications (ICC 2015), 2015.

[16] K. Dong, J. He, and W. Song, "Qoe-aware adaptive bitrate video streaming over mobile networks with caching proxy," in Computing, Networking and Communications (ICNC), 2015 International Conference on. IEEE, 2015, pp. 737-741.

[17] S. Xiang, M. Xing, L. Cai, and J. Pan, "Dynamic rate adaptation for adaptive video streaming in wireless networks," Signal Processing: Image Communication, vol. 39, pp. 305-315, 2015.

[18] H. Chenji and Z. J. Haas, "Enhancement of wireless bandwidth utilization through user's qoe," in Wireless Communications and Networking Conference (WCNC), 2015 IEEE. IEEE, 2015, pp. 2038-2043.

[19] A. Farshad, P. Georgopoulos, M. Broadbent, M. Mu, and N. Race, "Leveraging sdn to provide an in-network qoe measurement framework," in Computer Communications Workshops (INFOCOM WKSHPS), 2015 IEEE Conference on. IEEE, 2015, pp. 239-244.

[20] M. Mu, S. Steven, F. Arsham, N. Qiang, and R. Nicholas, "Userlevel fairness delivered: Network resource allocation for adaptive video streaming," in International Symposium on Quality of Service (IWQoS), 2015 IEEE/ACM Proceedings of. IEEE/ACM, 2015, pp. 239-244.

[21] A. Mansy, M. Fayed, and M. Ammar, "Network-layer fairness for adaptive video streams," in IFIP Networking Conference (IFIP Networking), 2015. IEEE, 2015, pp. 1-9.

[22] [Online]. Available: https://goo.gl/V4khZ5

[23] S. Sundaresan, N. Feamster, and R. Teixeira, "Locating throughput bottlenecks in home networks," in Proceedings of the 2014 ACM conference on SIGCOMM. ACM, 2014, pp. 351-352.

[24] R. M. Abuteir, A. Fladenmuller, and O. Fourmaux, "Sdn based architecture to improve video streaming in home networks," in Advanced Information Networking and Applications (AINA), 2016 IEEE International Conference on. IEEE, 2016.

[25] M. S. Seddiki, M. Shahbaz, S. Donovan, S. Grover, M. Park, N. Feamster, and Y.-Q. Song, "Flowqos: Qos for the rest of us," in Proceedings of the third workshop on Hot topics in software defined networking. ACM, 2014, pp. 207-208.

[26] A. Patro, S. Govindan, and S. Banerjee, "Observing home wireless experience through wifi aps," in Proceedings of the 19th annual international conference on Mobile computing \& networking. ACM, 2013, pp. 339350.

[27] [Online]. Available: https://goo.gl/rOZ73s

[28] [Online]. Available: https://goo.gl/DhwFdK

[29] R. K. Jain, D.-M. W. Chiù, and W. R. Hawe, "A quantitative measure of fairness and discrimination for resource allocation in shared computer systems," 1984.

[30] [Online]. Available: https://www.nsnam.org

[31] [Online]. Available: https://peach.blender.org/

[32] [Online]. Available: https://www.ffmpeg.org/

[33] [Online]. Available: https://gpac.wp.mines-telecom.fr

[34] S. Akhshabi, A. C. Begen, and C. Dovrolis, "An experimental evaluation of rate-adaptation algorithms in adaptive streaming over http," in Proceedings of the second annual ACM conference on Multimedia systems. ACM, 2011, pp. 157-168.

[35] T.-Y. Huang, R. Johari, N. McKeown, M. Trunnell, and M. Watson, "A buffer-based approach to rate adaptation: Evidence from a large video streaming service," in Proceedings of the 2014 ACM conference on SIGCOMM. ACM, 2014, pp. 187-198. 